\title{
Shock-capturing with natural high-frequency oscillations
}

\author{
Y. C. Zhou ${ }^{1}$, Yun $\mathrm{Gu}^{1}$ and G. W. Wei ${ }^{1,2, *, \dagger}$ \\ ${ }^{1}$ Department of Computational Science, National University of Singapore, Singapore 117543, Singapore \\ ${ }^{2}$ Department of Mathematics, Michigan State University, East Lansing, MI 48824, U.S.A.
}

\begin{abstract}
SUMMARY
This paper explores the potential of a newly developed conjugate filter oscillation reduction (CFOR) scheme for shock-capturing under the influence of natural high-frequency oscillations. The conjugate low- and high-pass filters are constructed based on the principle of the discrete singular convolution (DSC), a local spectral method. The accuracy and resolution of the DSC basic algorithm are accessed with a one-dimensional advection equation. Two Euler systems, the advection of an isotropic vortex flow and the interaction of shock-entropy wave are utilized to demonstrate the utility of the CFOR scheme. Computational accuracy and order of approximation are examined and compared with the literature. Some of the best numerical results are obtained for the shock-entropy wave interaction. Numerical experiments indicate that the CFOR scheme is stable, conservative and reliable for the numerical simulation of hyperbolic conservation laws. Copyright (c) 2003 John Wiley \& Sons, Ltd.
\end{abstract}

KEY WORDS: discrete singular convolution; conjugate filter oscillation reduction; shock capture; shock-entropy wave interaction

\section{INTRODUCTION}

The numerical difficulties of the non-linear hyperbolic conservation laws are of long standing and well known. Despite many efforts in the past several decades, attaining solutions that are free of spurious oscillations for compressible Euler equations involving steep gradient at discontinuities still remains a major problem in computational fluid dynamics (CFD). In particular, to construct a high-accuracy and high-resolution solution for a system involving the interaction of shock-turbulent boundary layer is a severe challenge due to its natural high-frequency components. Traditional schemes such as upwind, Riemann solver, approximate Riemann solver, random choice method and artificial viscosity method, are usually of low order in nature. More sophisticated approaches, such as total variation diminishing (TVD), essentially non-oscillatory (ENO) [1], weighted essentially non-oscillatory (WENO), characteristic-based-split (CBS) [2] and discontinuous Galerkin schemes [3] are proposed.

*Correspondence to: G. W. Wei, Department of Mathematics, Michigan State University, East Lansing, MI 48824, U.S.A.

†E-mail: wei@math.msu.edu

Contract/grant sponsor: National University of Singapore

Copyright (c) 2003 John Wiley \& Sons, Ltd.

Received October 2001

Revised 15 July 2002 
Generally, these shock-capturing schemes are shown to be very successful in many applications.

Recently, it has been pointed out [4] that the use of a sixth-order accurate shock-capturing scheme in the entire computational domain leads to a significant damping of turbulent fluctuations. Garnier et al. [5] found that in the framework of freely decaying turbulence, the numerical dissipation of high-order accurate shock-capturing schemes masks the effect of a subgrid-scale model. Therefore, alternative approaches are of pressing desirable for many practical applications. The use of filters is one of important alternative approaches which can overcome the problem of excessive numerical dissipation in many sophisticated shockcapturing schemes. Engquist et al. [6] proposed a set of non-linear filters which discriminate and suppress the dispersive wiggles in the basic solution. Recently, Garnier et al. [7] have reported the use of the non-linear dissipation components in some high-order shock-capturing schemes, such as the ENO and WENO, as filters. With appropriate sensors, these ENO/WENO filters are shown to effectively improve the resolution of density waves, entropy waves and stochastic turbulent fluctuations. Indeed, in the case of direct simulation of turbulence, typical successful numerical approaches are compact schemes [8] or spectral methods for their capability of resolving multiscale features in turbulent fluctuations. However, spectral methods are notorious for their Gibbs' oscillations near the discontinuity, while compact schemes that are commonly used for shock capturing are of third- or at most fifth-order. Therefore, it is highly desirable to have methods that are of (arbitrary) high accuracy for resolving highfrequency waves and stochastic turbulent fluctuations, and are capable of shock capturing without excessive numerical dissipation.

Conjugate filter oscillation reduction (CFOR) $[9,10]$ is one of such schemes newly developed for solving practical problems. The CFOR scheme is constructed based on the discrete singular convolution (DSC) algorithm [11-13] for the numerical computation of singular convolutions. The theoretical foundation of the DSC algorithm is the theory of distributions and the theory of wavelet analyses. In its appropriate form, the DSC algorithm is a local spectral method. It provides a unified approach to conventional local and global methods and has controllable accuracy for the numerical solution of differential equations. The essential idea in the CFOR scheme is to use conjugate (DSC) low-pass filters to remove spurious oscillations generated by conjugate (DSC) high-pass filters which are implemented for the numerical approximation of differentiation operators. Conjugate filters are constructed by using DSC kernels and are optimal in the sense that they have similar degrees of regularity, accuracy, frequency bandwidth and computational supports. Such a feature makes the DSC low-pass filter differ from Ghosal's dealiasing filters [14] in the sense that one could continuously adjust the 'degree' of the conjugation, and also differ from Fedioun's interpolation filter [15] in the sense that there is no ripples in the passband of the low-pass filters. The CFOR scheme has been successfully applied to shock-capturing in association with Burgers' equation, one- and two-dimensional (2D) Euler systems including the Sod and Lax problems, and a Mach 3 flow past a wind tunnel with a step. The most promising feature of the CFOR scheme is that, the approach has a controllable order of approximation for shock-capturing under specific situations. The objective of the present work is to explore the utility and limitation of the CFOR scheme in dealing with the problem of shock-high-frequency entropy wave interaction, which is very challenging because conventional methods encounter the difficulty of either insufficient accuracy or excessive numerical damping. It is believed that a better understanding of the CFOR scheme is of importance to the development of high-accuracy 
and low-dissipation schemes for the numerical solution of more challenging problems, such as shock-turbulence interaction.

This paper is organized as follows. A brief retrospection is given to the DSC algorithm and the CFOR scheme in Section 2. Section 3 is devoted to the numerical experiments of a 1D advection equation and two Euler systems. The last example is designed to test the capability of resolving shock from high-frequency entropy waves. A conclusion ends the paper.

\section{THE DSC ALGORITHM AND CFOR SCHEME}

\subsection{DSC filters}

Singular convolutions occur commonly in science and engineering. Discrete singular convolution (DSC) is an effective approach for the numerical realization of singular convolutions. There are many detailed descriptions about the discrete singular convolution in the literature [11-13]. The introduction in Reference [11] is recommended for its theoretical underpinning and approximation philosophy. For the sake of integrity and convenience, a brief review of the DSC algorithm is given before describing the CFOR scheme.

In the context of distribution theory, a singular convolution can be defined by

$$
F(t)=(T * \eta)(t)=\int_{-\infty}^{\infty} T(t-x) \eta(x) \mathrm{d} x
$$

where $T$ is a singular kernel and $\eta(x)$ is an element of the space of test functions. Interesting examples include singular kernels of Hilbert (and Abel) type and delta type. The former plays an important role in the theory of analytical functions, processing of analytical signals, theory of linear responses and Radon transform. Since delta type kernels are the key element in the approximation theory and the numerical solution of differential equations, we focus on the singular kernels of delta type

$$
T(x)=\delta^{(q)}(x) \quad(q=0,1,2, \ldots)
$$

where superscript $(q)$ denotes the $q$ th-order 'derivative' of the delta distribution, $\delta(x)$, with respect to $x$, which should be understood as generalized derivatives of distributions. When $q=0$, the kernel, $T(x)=\delta(x)$, is important for the interpolation of surfaces and curves, including applications to the design of engineering structures. For hyperbolic conservation laws and Euler systems, two special cases, $q=0$ and 1 are involved, whereas for the full NavierStokes equations, the case of $q=2$ will be also invoked. Because of its singular nature, the singular convolution of Equation (1) cannot be directly used for numerical computations. In addition, the restriction to the test function is too strict for most practical applications. To avoid the difficulty of using singular expressions directly in numerical computations, we consider a discrete singular convolution which provides appropriate approximations to the original distribution

$$
f^{(q)}(x) \approx \sum_{k} \delta_{\Delta}^{(q)}\left(x-x_{k}\right) f\left(x_{k}\right)
$$

where $\delta_{\Delta}^{(q)}\left(x-x_{k}\right)$ are approximations to $\delta^{(q)}\left(x-x_{k}\right)$ and are designed for being used in (discrete) summations. Here, $\left\{x_{k}\right\}$ is an appropriate set of discrete points centred around the 
point $x$ and $\Delta$ is the grid spacing. Depending on the mathematical properties of the kernel, $\delta_{\Delta}$, the restriction on the $f\left(x_{k}\right)$ can be relaxed to include many common-occurring functions. A variety of candidates are available for $\delta_{\Delta}$ in the literature. Among these examples, Shannon's delta kernel is of particular interesting

$$
\delta_{\Delta}(x)=\sin (\alpha x) / \pi x
$$

Shannon's kernels are a delta sequence and thus provide approximations to the delta distribution

$$
\lim _{\alpha \rightarrow 0}<\frac{\sin (\alpha x / \Delta)}{\pi x}, \quad \eta(x)>=\eta(0)
$$

Shannon's kernel has been widely used in information theory, signal and image processing because the Fourier transform of Shannon's kernel is an ideal low-pass filter. However, the usefulness of Shannon's kernel is limited by the fact that it has a slow-decaying oscillatory tail proportional to $1 / x$ in the co-ordinate domain. For signal processing, Shannon's kernel is an infinite impulse response (IIR) low-pass filter. Therefore, when truncated in computational applications, its Fourier transform contains evident oscillations. A cure to this problem is to regularize Shannon's kernel with a Gaussian

$$
\delta_{\sigma, \Delta}(x)=\frac{\sin (\pi x / \Delta)}{\pi x} \mathrm{e}^{-x^{2} / 2 \sigma^{2}}, \quad \sigma>0
$$

Since $\mathrm{e}^{-x^{2} / 2 \sigma^{2}}$ is a Schwartz class function, it makes regularized Shannon's kernel applicable to tempered distributions. Moreover, as the regularized kernels decay very fast in the space domain, they can be utilized as finite impulse response (FIR) low-pass filters. Their oscillation in the Fourier domain is dramatically reduced and effectively controlled.

For sequences of the delta type, an interpolating algorithm sampling at the Nyquist frequency, $\pi / \Delta$, has an advantage over a non-interpolating discretization. Therefore, on a uniform grid, the regularized Shannon's kernel is discretized as

$$
\delta_{\sigma, \Delta}\left(x-x_{k}\right)=\frac{\sin \left[(\pi / \Delta)\left(x-x_{k}\right)\right]}{\pi / \Delta\left(x-x_{k}\right)} \mathrm{e}^{-\left(x-x_{k}\right)^{2} / 2 \sigma^{2}}
$$

The regularized kernel $\delta_{\sigma, \Delta}(x)$ corresponds to a family of FIR low-pass filters, each with a different compact support, according to $\sigma / \Delta$, in the co-ordinate domain. Its $q$ th-order derivative is given by analytical differentiation

$$
\delta_{\sigma, \Delta}^{(q)}\left(x-x_{k}\right)=\left(\frac{\mathrm{d}}{\mathrm{d} x}\right)^{(q)} \frac{\sin \left[(\pi / \Delta)\left(x-x_{k}\right)\right]}{\pi / \Delta\left(x-x_{k}\right)} \mathrm{e}^{-\left(x-x_{k}\right)^{2} / 2 \sigma^{2}}
$$

In this work, $\delta_{\sigma, \Delta}^{(q)}(x),(q=0,1, \ldots)$ are referred as a family of 'conjugate filters', as they are derived from one generating function and consequently have a similar degree of regularity, smoothness, time-frequency localization, effective support and bandwidth.

In application, optimal results are usually obtained if the window size $\sigma$ varies as a function of the central frequency $\pi / \Delta$, such that $r=\sigma / \Delta$ is a parameter chosen in computations. Both interpolation and differentiation are realized by the following convolution algorithm:

$$
f^{(q)}(x) \approx \sum_{k=-W}^{k=W} \delta_{\sigma, \Delta}^{(q)}\left(x-x_{k}\right) f\left(x_{k}\right) \quad(q=0,1,2, \ldots)
$$


where $2 W+1$ is the computational bandwidth, or effective kernel support, which is usually smaller than the entire computational domain, [a,b]. Expressions of $\delta_{\sigma, \Delta}^{(q)}(x)$ with $q=0,1$ are given as

$$
\begin{gathered}
\delta_{\sigma, \Delta}(x)= \begin{cases}\frac{\sin (\pi x / \Delta) \exp \left(-x^{2} / 2 \sigma^{2}\right)}{\pi x / \Delta} & (x \neq 0) \\
1 & (x=0)\end{cases} \\
\delta_{\sigma, \Delta}^{(1)}(x)= \begin{cases}\frac{\cos (\pi x / \Delta) \exp \left(-x^{2} / 2 \sigma^{2}\right)}{x}-\frac{\sin (\pi x / \Delta) \exp \left(-x^{2} / 2 \sigma^{2}\right)}{\pi x^{2} \Delta} & (x \neq 0) \\
-\frac{\sin (\pi x / \Delta) \exp \left(-x^{2} / 2 \sigma^{2}\right)}{\pi \sigma^{2} / \Delta} & (x=0) \\
0 & \end{cases}
\end{gathered}
$$

Expressions of higher-order derivatives for $\delta_{\sigma, \Delta}(x)$ can be found elsewhere [13]. Rigorous mathematical analysis of the algorithm in Sobolev spaces is accounted in Reference [16].

\subsection{The CFOR scheme}

Consider 2D Euler equations for gas dynamics having the conservation form of

$$
U_{t}+F(U)_{x}+G(U)_{y}=0
$$

with

$$
U=\left(\begin{array}{c}
\rho \\
\rho u \\
\rho v \\
E
\end{array}\right) ; \quad F(U)=\left(\begin{array}{c}
\rho u \\
\rho u^{2}+p \\
\rho u v \\
u(E+p)
\end{array}\right) ; \quad G(U)=\left(\begin{array}{c}
\rho v \\
\rho u v \\
\rho v^{2}+p \\
v(E+p)
\end{array}\right)
$$

where, $\rho, u, v, p$ and $E$ denote the density, the velocities in $x$-and $y$-directions, the pressure and the total energy per unit mass $E=\rho\left(e+\left(u^{2}+v^{2}\right) / 2\right)$, respectively. Here, $e$ is the specific internal energy. For an ideal gas with constant specific heat ratio $(\gamma=1.4)$ considered here, one has $e=p /(\gamma-1) \rho$.

Let denote the grid point $(i \Delta x, j \Delta y)$ as $\left(x_{i}, y_{j}\right)$ and the spatial discretizations of $F(U)$ and $G(U)$ at the point $\left(x_{i}, y_{j}\right)$ as $F\left(U_{i, j}\right)$ and $G\left(U_{i, j}\right)$. Their spatial derivatives $F(U)_{x}$ and $G(U)_{y}$ are approximated by the DSC high-pass filters according to Equation (3), i.e.

$$
\begin{aligned}
F\left(U_{i, j}\right)_{x} & =\sum_{k=i-W}^{i+W} \delta_{\sigma, \Delta_{x}}^{(1)}\left(x_{i}-x_{k}\right) F\left(U_{k, j}\right) \\
G\left(U_{i, j}\right)_{y} & =\sum_{k=j-W}^{j+W} \delta_{\sigma, \Delta_{y}}^{(1)}\left(y_{j}-y_{k}\right) G\left(U_{i, k}\right)
\end{aligned}
$$

Here we assume a uniform mesh in both $x$ - and $y$-direction with the grid intervals being $\Delta_{x}$ and $\Delta_{y}$, respectively. The accuracy of the DSC algorithm is controllable [13]. 
Although there is no rigorous proof about whether the standard fourth-order Runge-Kutta (RK-4) scheme is TVD, it is still one of the most widely used temporal schemes for hyperbolic conservation laws. The RK-4 is adopted in the present work and it takes the following form in our problems:

$$
\begin{aligned}
k_{1} & =-F\left(U_{i, j}^{n}\right)_{x}-G\left(U_{i, j}^{n}\right)_{y} \\
k_{2} & =-F\left(U_{i, j}^{n}+\frac{\Delta t}{2} k_{1}\right)_{x}-G\left(U_{i, j}^{n}+\frac{\Delta t}{2} k_{1}\right)_{y} \\
k_{3} & =-F\left(U_{i, j}^{n}+\frac{\Delta t}{2} k_{2}\right)_{x}-G\left(U_{i, j}^{n}+\frac{\Delta t}{2} k_{2}\right)_{y} \\
k_{4} & =-F\left(U_{i, j}^{n}+\Delta t k_{3}\right)_{x}-G\left(U_{i, j}^{n}+\Delta t k_{3}\right)_{y} \\
U_{i, j}^{n+1} & =U_{i, j}^{n}+\frac{\Delta t}{6}\left[k_{1}+2 k_{2}+2 k_{3}+k_{4}\right]
\end{aligned}
$$

The approximation of $F(U)_{x}$ and $G(U)_{y}$ by using the DSC high-pass filters in Equations (14) and (15), together with the RK-4 scheme (16)-(20), provides a basic scheme for the numerical integration of the Euler system, Equation (12). No additional effort is required if the problem under consideration does not involve discontinuity. Otherwise, an additional filtering can be implemented to suppress spurious oscillations.

The conjugation, resolution and variation of these filters can be effectively justified with Fourier analysis, which has been widely used to quantify compact schemes [8] and some general numerical schemes for hyperbolic equations [17]. The frequency responses of conjugate low- and high-pass filters [18] are illustrated in Figure 1. As aforementioned, the conjugate filters are constructed from the same generating function (7). It can be seen that below $0.7 \pi / \Delta$, all the conjugate filters are highly accurate. However, in the high-frequency region, the frequency responses of both the low-pass filter and first-order high-pass filter are serious under estimating, whereas the frequency response of the second-order high-pass filter is over estimating. The error in the high-frequency response is harmless for numerical problems involving only low frequency components. However, in the case of shock and discontinuity, the solution contains much high-frequency component, the error in the high-frequency response will be accumulated and amplified during the time integration, and leads to spurious oscillations. This observation motivates us to use the conjugate low-pass filter to appropriately eliminate most of the high-frequency response produced by the conjugate high-pass filters. As a result, the solution generated by conjugate filters is reliable for the frequency below the effective bandwidth of the filters. The effective bandwidth or frequency cut-off is controlled by the choice of the DSC parameter $r=\sigma / \Delta$, for a given $\Delta$. The CFOR scheme is implemented via the following two-step procedure:

$$
\begin{aligned}
& \hat{U}_{i, j}^{n+1}=H\left(U_{i, j}^{n}\right) \\
& U_{i, j}^{n+1}=L\left(\hat{U}_{i, j}^{n+1}\right)
\end{aligned}
$$




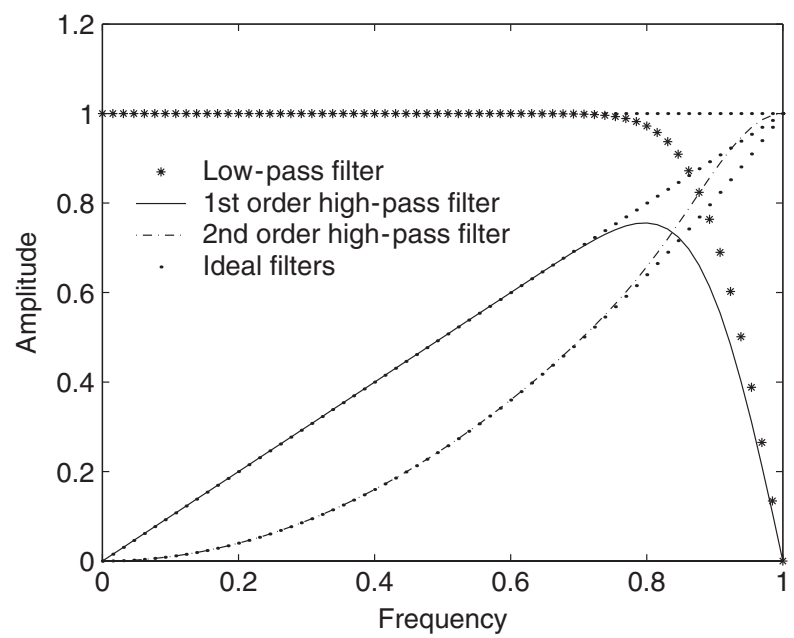

Figure 1. Frequency responses of the conjugate DSC filters (in the unit of $\pi / \Delta$ ), The maximum amplitude of the filters is normalized to the unit.

where $H\left(U_{i, j}^{n}\right)$ is the high-pass filtering process numerically defined by Runge-Kutta scheme (16)-(20). Here, $L$ is the DSC low-pass filtering as shown in Equation (3) with $q=0$. This interpolative low-pass filter is implemented through prediction (in which the variables on the grid are interpolated to the middle points of the cells) and restoration (in which the variables on the grid are restored from their values at the middle points of the cells) [10]. Such prediction-restoration is carried out in each dimension. In either direction, this can be expressed by

$$
\begin{aligned}
U_{i+1 / 2}^{n}= & \sum_{k=-W}^{-1} U_{i+k+1}^{n} \delta_{\sigma, \Delta}\left(\left(k+\frac{1}{2}\right) \Delta\right)+\sum_{k=1}^{W} U_{i+k}^{n} \delta_{\sigma, \Delta}\left(\left(k-\frac{1}{2}\right) \Delta\right) \\
& \quad \text { or prediction } \\
U_{i}^{n}= & \sum_{k=-W}^{-1} U_{i+k+1 / 2}^{n} \delta_{\sigma, \Delta}\left(\left(k+\frac{1}{2}\right) \Delta\right)+\sum_{k=1}^{W} U_{i+k-1 / 2}^{n} \delta_{\sigma, \Delta}\left(\left(k-\frac{1}{2}\right) \Delta\right)
\end{aligned}
$$

for restoration

In the above two-step procedure (21)-(22), the second step, i.e. low-pass conjugate filtering (22) is controlled (turned on or turned off) by a sensor. To construct sensors, we defined a high-frequency measure $M$. Upon the increment $\Delta M$ exceeding a prescribed threshold $\zeta$, the low-pass filtering process is carried out and $L\left(\hat{U}_{i, j}^{n+1}\right)$ is the solution $U_{i, j}^{n+1}$ at the new time step $n+1$; otherwise, no low-pass filter will be exerted to $\hat{U}_{i, j}^{n+1}$ and the latter is admitted as the solution $U_{i, j}^{n+1}$ at the $n+1$ time step. The high-frequency measure $M$ is defined via a multiscale wavelet transform of a set of discrete function values at time $t_{n}$ as

$$
\left\|M^{n}\right\|=\sum_{m}\left\|M_{m}^{n}\right\|
$$


where $\left\|M_{m}^{n}\right\|$ is given by a convolution with a wavelet $\psi_{m j}$ of scale $m$

$$
\left\|M_{m}^{n}\right\|=\sum_{k}\left|\sum_{j} \psi_{m j}\left(x_{k}\right) u^{n}\left(x_{j}\right)\right|
$$

Such a definition can be further illustrated by one of its special case - the TVD sensor, which by definition, is

$$
\left\|M^{n}\right\|=\sum_{i}\left|u_{i+1}^{n}-u_{i}^{n}\right|
$$

and can be recognized as the convolution with the Haar wavelet of a single scale. This TVD sensor seems to be the simplest one among a number of sensors which can be used in the present scheme. The choice of the threshold $\zeta$ for the high-frequency measure $\Delta M=\left\|M^{n+1}\right\|-$ $\left\|M^{n}\right\|$ depends on the nature of the problem under study and is in the range from 0.001 to 0.002 .

The separation of the basic spatial discretization (the high-pass filtering) and the postprocessing (low-pass filtering) in the proposed shock-capturing scheme makes it possible to focus on the design of a set of versatile and efficient filters. Such an approach has a few advantages. First, the basic DSC algorithm is a local method but it can be as accurate as a spectral method, as is shown in the next section. Therefore, the CFOR scheme has controllable accuracy via the choice of filter parameters. Secondly, the filtering is adaptive and is applied at most once per iteration circle, comparing with pointwise treatment at each grid point in many other schemes. Therefore, there is a potential increase in the computational efficiency by a mature CFOR code. Finally, the implementation of the conjugate low-pass filter and filter parameters are easily controlled. Hence, a physical high-frequency wave can be effectively distinguished from the shock induced spurious oscillations in the present approach.

\section{RESULTS AND DISCUSSIONS}

In this section, we justify the differentiation accuracy of the DSC algorithm with a linear advection equation. Then, we examine the utility and explore limitation of the proposed CFOR scheme by using two other benchmark numerical problems, the $2 \mathrm{D}$ advection of an isentropic vortex $[1,12]$ and the interaction of shock and entropy wave [1]. The first Euler system is designed to quantitatively access the phase and amplitude errors [5] of the CFOR scheme in handling 2D Euler problems. To maintain a small error in both the phase and amplitude is particularly desirable for a scheme to handle shock-entropy wave interaction and many other aerodynamic problems. Moreover, extensive numerical data are available for this problem and a comparison with many other shock-capturing schemes, such as the ENO and WENO, is readily possible. The second problem is a standard test for the numerical ability of treating the interaction of high-frequency entropy wave and shock. It is a severe challenge for most existing shock-capturing methods due to its highly oscillatory nature. Numerical results can be objectively evaluated by a quantitative criterion obtained from a linear analysis. Parameters $W=32$ and $r=3.2$ are used for all the high-pass filtering and low-pass prediction. For the low-pass restoration, $r=3.2$ is used in the evolution of isentropic vortex, while $r$ values of 1.9-2.1 are used in the interaction of shock and high-frequency entropy waves. 
Table I. $L_{2}$ error for solving the $1 \mathrm{D}$ advection equation $(N=400)$.

\begin{tabular}{lccccc}
\hline$r$ & $k=1$ & $k=2$ & $k=3$ & $k=4$ & $k=5$ \\
\hline 3.2 & $1.93 \mathrm{E}-13$ & $7.38 \mathrm{E}-12$ & $4.42 \mathrm{E}-9$ & $3.08 \mathrm{E}-6$ & $8.24 \mathrm{E}-4$ \\
4.2 & $8.85 \mathrm{E}-13$ & $5.64 \mathrm{E}-12$ & $3.84 \mathrm{E}-11$ & $1.67 \mathrm{E}-10$ & $1.28 \mathrm{E}-7$ \\
\hline
\end{tabular}

\subsection{Linear advection equation}

The linear equation we considered here is

$$
\frac{\partial u}{\partial t}+\frac{\partial u}{\partial x}=0
$$

whose initial condition is denoted by $u_{0}=u(x, 0)$.

To closely observe the magnitude response of the numerical scheme to particular components, we choose the initial condition of the form

$$
u_{0}=(\sin (2 \pi k x)-1) \mathrm{e}^{-\left(x-x_{0}\right)^{2} /\left(2 \sigma^{2}\right)}
$$

which is the linear combination of two Gaussian waves; one is centred around the zero wavenumber while another is modulated by a sine wave whose frequency is adjustable. We choose $\sigma=1.0$ so that the tails of Gaussian envelopes decay to near zero at the ends of our computation domain of $[10 \times 10]$. The equation is integrated up to $t=20$ with the time increment of $\Delta t=10^{-4}$ for all cases. For the periodic boundary condition we employed, the exact solution at any time can be obtained easily and the $L_{2}$ error against the exact solution is listed in Table I for $k$ values from 1 to 5 .

It is apparent that the error grows significantly with the increase of the wavenumber $k$, which determines the location of the sine wave in the frequency representation, as shown in Figure 2. The derivation of the DSC differentiation operator from the ideal differentiation operator at those locations in turn determines the spatial differentiation error. Therefore, it is not surprising that errors for $k=1$ are as small as $10^{-13}$ while errors for $k=5$ are much larger. The increase of $r$ from 3.2 to 4.2 results in a dramatic improvement on the accuracy of the DSC differentiation operator, as indicated by the right shifting of four square markers in the figure and by the change in the error for the cases of $k=3,4$ and 5 . In the succeeding computations, however, we still take $r=3.2$ in the derivative approximation, since the effective frequency range is also limited by the low-pass filters in practical computations. The typical value of $r$ for low-pass filters ranges between 1.9 and 2.1, as mentioned at the beginning of this section. Moreover, Yang et al. demonstrated [18] that the further increase in $r$ would lead to the decrease of accuracy for low components.

It is also necessary to investigate the computational cost of the DSC scheme since it employs a much wider stencil than the usual finite difference and finite volume methods. To this end, we choose a conservative centred fourth-order scheme (C4) of Reference [7], where it was used as the basic scheme of a new shock-capturing method. The $\mathrm{C} 4$ scheme takes the following form in computing the numerical flux at point $i+\frac{1}{2}$

$$
f(u)_{i+1 / 2}=\frac{1}{12}\left(-u_{i+2}+7 u_{i+1}+7 u_{i}-u_{i-1}\right)
$$



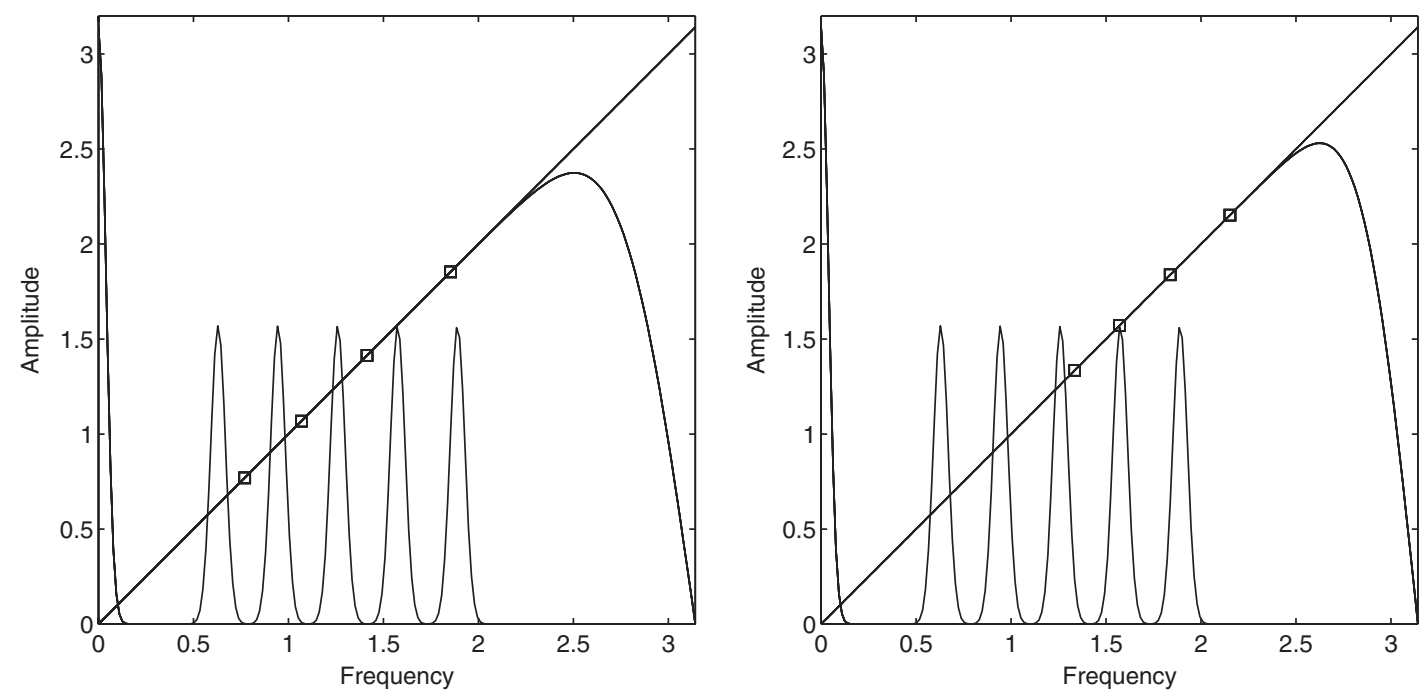

Figure 2. Frequency responses of the DSC differential operator for the first-order derivative and initial conditions with different frequency $k$. Left: $r=3.2$; Right: $r=4.2$. Four cases $k=2,3,4,5$ are plotted in each figure. Four square markers in each plot denote the locations beyond which the magnitude difference between the DSC filter and the ideal filter will exceed $10^{-13}, 10^{-10}$, $10^{-7}$, and $10^{-4}$, from the left to right, respectively.

Table II. Comparison of the DSC and C4 schemes for solving the 1D advection equation $(k=1)$.

\begin{tabular}{lrrr}
\hline Scheme & \multicolumn{1}{c}{$N$} & $L_{2}$ error & CPU \\
\hline C4 & 400 & $4.85 \mathrm{E}-2$ & 1.8 \\
& 1000 & $1.27 \mathrm{E}-3$ & 4.4 \\
& 2000 & $7.94 \mathrm{E}-5$ & 9.0 \\
DSC & 20000 & $9.95 \mathrm{E}-9$ & 113.0 \\
& 400 & $1.93 \mathrm{E}-13$ & 13.2 \\
\hline
\end{tabular}

The results for the $\mathrm{C} 4$ scheme are tabulated in Table II. It is seen that an extra fine mesh and thus a much larger CPU time are required for the $\mathrm{C} 4$ scheme to achieve comparable accuracy of the DSC algorithm with a much coarser mesh. Therefore, for a given grid system, using high order schemes such as the DSC can significantly reduce the computational cost.

\subsection{Isentropic vortex}

To quantitatively analyse the performance of the proposed CFOR scheme, the advection of an isotropic vortex in a free stream is computed. As the exact solution of the problem is available, it is an excellent benchmark for accessing the accuracy and stability of shockcapturing schemes and has been previously considered by many researchers $[1,7]$.

Consider a mean flow of $\left(\rho_{\infty}, u_{\infty}, v_{\infty}, P_{\infty}, T_{\infty}\right)=(1,1,1,1,1)$ with a periodic boundary condition in both directions. At $t_{0}$, the flow is perturbed by an isentropic vortex $\left(u^{\prime}, v^{\prime}, T^{\prime}\right)$ centred 
at $\left(x_{0}, y_{0}\right)$, having the form of

$$
\begin{aligned}
u^{\prime} & =-\frac{\lambda}{2 \pi}\left(y-y_{0}\right) \mathrm{e}^{\eta\left(1-r^{2}\right)} \\
v^{\prime} & =\frac{\lambda}{2 \pi}\left(x-x_{0}\right) \mathrm{e}^{\eta\left(1-r^{2}\right)} \\
T^{\prime} & =-\frac{(\gamma-1) \lambda^{2}}{16 \eta \gamma \pi^{2}} \mathrm{e}^{2 \eta\left(1-r^{2}\right)}
\end{aligned}
$$

Here, $r=\sqrt{\left(x-x_{0}\right)^{2}+\left(y-y_{0}\right)^{2}}$ is the distance to the vortex centre; $\lambda$ is the strength of the vortex and $\eta$ is a parameter determining the gradient of the solution, and is unity in this study. Note that for an isentropic flow, relations $p=\rho^{\gamma}(\gamma=1.4)$ and $T=p / \rho$ are valid. Therefore, the perturbation in $\rho$ is required to be

$$
\rho=\left(T_{\infty}+T^{\prime}\right)^{1 /(\gamma-1)}=\left[1-\frac{(\gamma-1) \lambda^{2}}{16 \eta \gamma \pi^{2}} \mathrm{e}^{2 \eta\left(1-r^{2}\right)}\right]^{1 /(\gamma-1)}
$$

For a comparison with the existing literature [7], the computational domain is chosen as $[0,10] \times[0,10]$ with the centre of the vortex being initially located at $\left(x_{0}, y_{0}\right)=(5,5)$, the geometrical centre of the computational domain. Two experiments are performed in this study with $\eta=1$ and $\lambda=0.5$. One is to examine the accuracy of the CFOR scheme and to compare with the available literature. The other is to investigate the stability and performance of the CFOR scheme for long-time integrations. For the first experiment, we compute the density profile up to $t=2$ using five sets of meshes $\left(N=N_{x}=N_{y}=20,40,80,160,320\right)$ which are selected by Garnier et al. [7]. In the present computations, the CFL number is chosen as 0.5 for a comparison with previous results [7].

Two error measures, $L_{1}$ and $L_{2}$, are used in this study. They are defined [7] by

$$
\begin{aligned}
& L_{1}=\frac{1}{(N+1)^{2}} \sum_{i=0}^{N} \sum_{j=0}^{N}\left|f_{i, j}-\bar{f}_{i, j}\right| \\
& L_{2}=\frac{1}{(N+1)} \sqrt{\sum_{i=0}^{N} \sum_{j=0}^{N}\left|f_{i, j}-\bar{f}_{i, j}\right|^{2}}
\end{aligned}
$$

where $f$ is the numerical result and $\bar{f}$ the exact solution (Note that they are not the standard definitions). The CFOR errors for the density with respect to the exact solution are listed in Tables III and IV. Highly accurate results are obtained, as shown by the tables. Obviously, the proposed scheme is much more accurate than any other scheme listed in the tables, which are reported by Garnier et al. [7]. Remarkably, the CFOR scheme is from 4 to 5 orders more accurate than other schemes when $N=80$.

As the spatial discretization of the CFOR scheme is extremely accurate, some of the present results computed at $\mathrm{CFL}=0.5$ might be limited by the CFL number, which was optimized according to various schemes given in Reference [7]. This is indeed the case. The CFOR results computed at $\mathrm{CFL}=0.01$ are generally more accurate than those obtained at $\mathrm{CFL}=0.5$ 


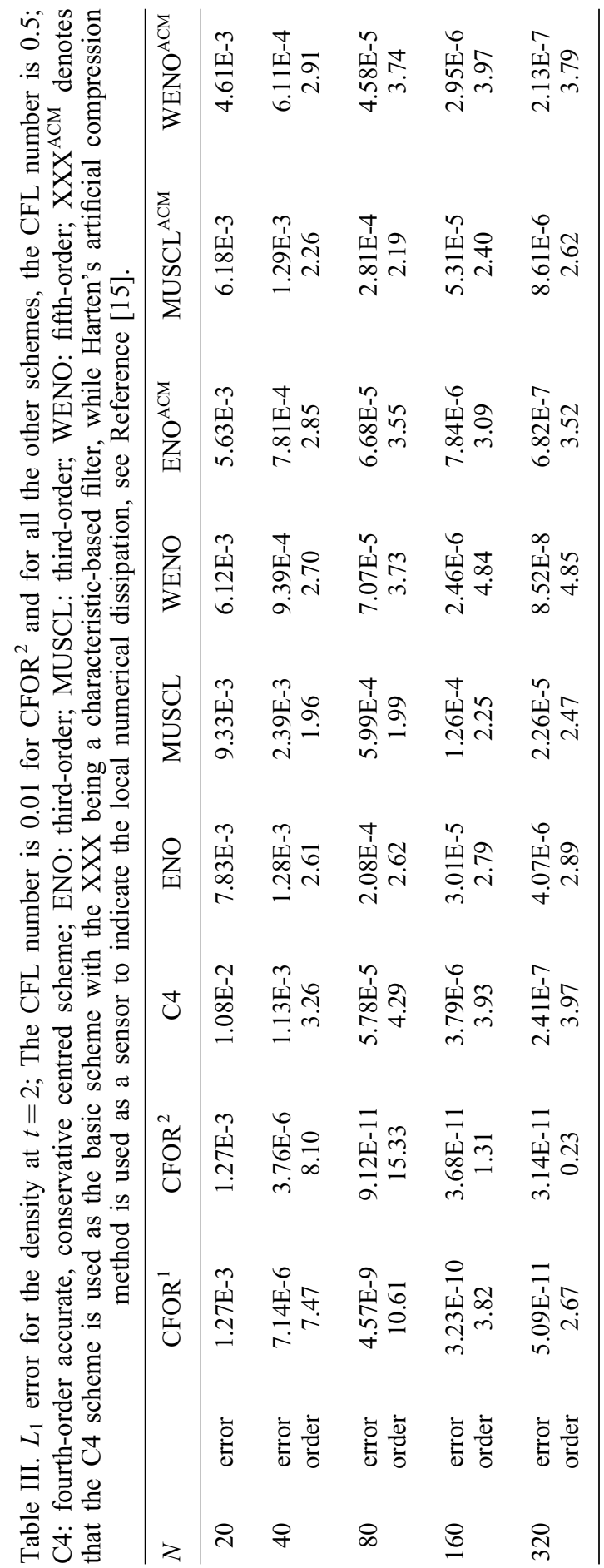




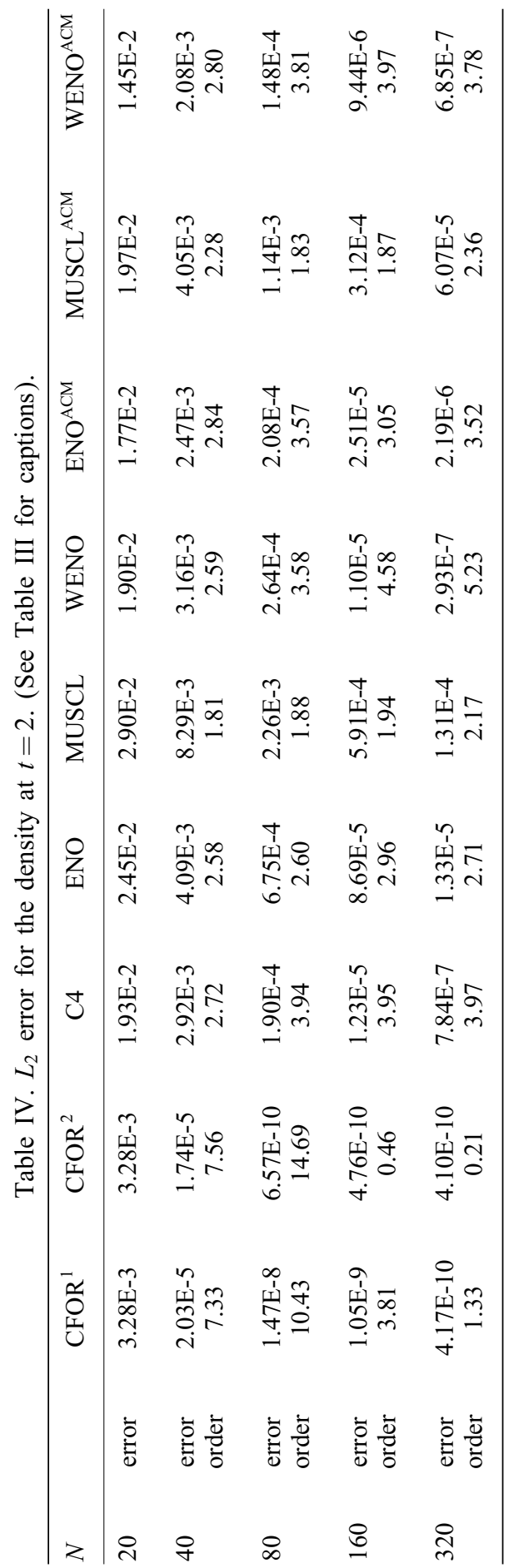




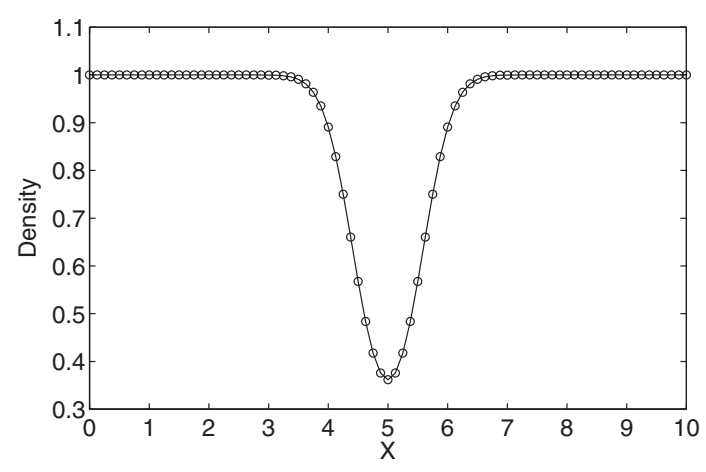

(a)

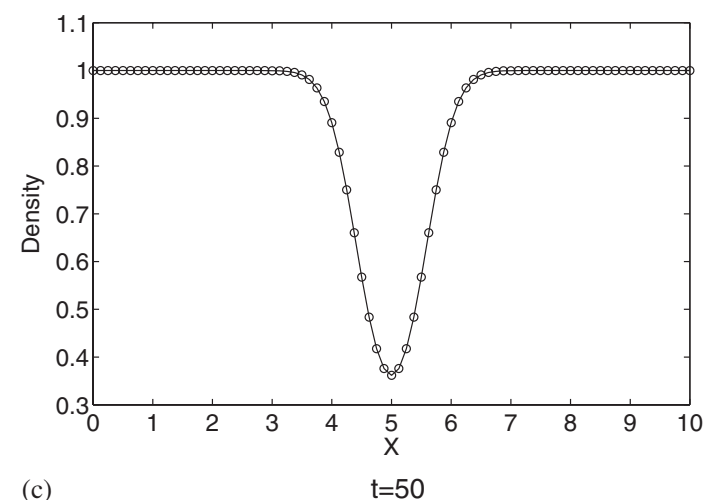

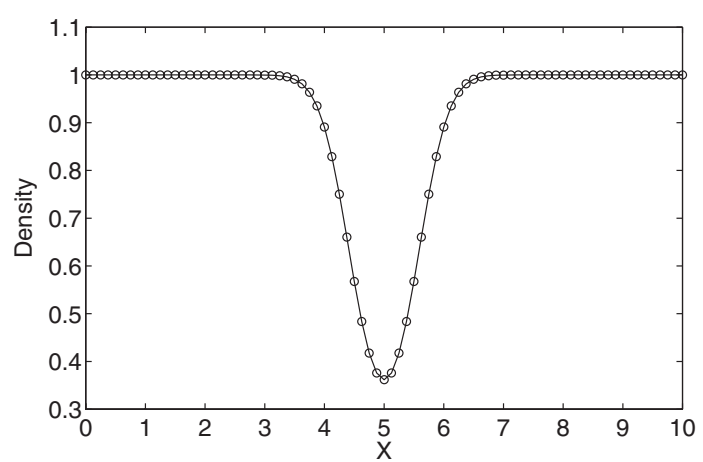

(b)

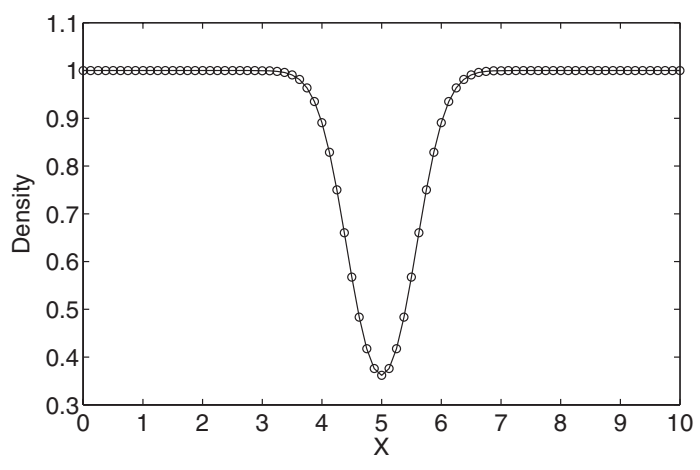

(d)

$t=100$

Figure 3. Density profiles in horizontal cutting at four times. Solid line is the exact profile and circle dots denote the numerical results.

as shown in Tables III and IV. Note that the accuracy of the CFOR scheme increases dramatically when the mesh is refined from 40 grid points to 80 grid points, with the numerically computed approximation order being more than 15. Therefore, the CFOR scheme has the feature of spectral-like methods. Obvious, due to its extremely high accuracy, the CFOR can be used for large scale simulations without resorting to a very large mesh as required by low order schemes.

Our next numerical experiment concerns the performance of the CFOR scheme for the long-time integration, which poses a severe challenge to the stability and conservation of the discretization scheme [1]. The solution of $\rho$ is sampled at $t=2,10,50$ and 100, with the grid spacing of $\Delta x=\Delta y=0.125$ and $C F L=0.5$. In Figure 3, we show the horizontal line cut through the centre of the vortex for the density $\rho$. Obviously, there is no visual deviation between the computed result and the exact one. Errors listed in Table V further confirm that the present scheme is accuracy, free of excessive dissipation and reliable.

Since there is no presence of shock in this case, the low-pass filter originally designed to suppress dispersive wiggles might appear useless. In this experiment, it is found that the DSC algorithm on its own can already provide excellent results if the integration time is 
Table V. Errors for the density at different times $(\mathrm{CFL}=0.5, N=80)$.

\begin{tabular}{lcccc}
\hline Time & 2 & 10 & 50 & 100 \\
\hline$L_{1}$ & $4.56 \mathrm{E}-9$ & $1.77 \mathrm{E}-8$ & $4.27 \mathrm{E}-8$ & $8.90 \mathrm{E}-8$ \\
$L_{2}$ & $1.47 \mathrm{E}-8$ & $4.95 \mathrm{E}-8$ & $1.44 \mathrm{E}-7$ & $3.01 \mathrm{E}-7$ \\
\hline
\end{tabular}

sufficiently small. Thus, the conjugate low-pass filter does not need to be activated during an initial time period. However, as the time progresses, errors would accumulate rapidly and the computation could become unstable if the low-pass filter were not used to effectively control the dramatical non-linear growth of the errors. Therefore, the CFOR scheme is very robust for the treatment of this problem. As shown in Table V and Figure $3(t=100)$, the long time simulation results are very stable. The vortex core is well conserved and the accuracy is extremely high. These results indicate that the CFOR scheme is highly accurate, stable and conservative for the long-time integration of Euler systems. It is a potential approach for the numerical integration of hyperbolic conservation laws. Its ability for shock-capturing is examined in the next subsection.

\subsection{Interaction of shock and high-frequency entropy wave}

The interaction of shock and high-frequency entropy wave is a standard test problem [1] for benchmarking potential high-order shock-capturing methods. The problem is significant due to its relevant to the interaction of shock and turbulence. A Mach 3 right-moving shock interacts with a small amplitude entropy wave. The computation domain is taken as $[0,5]$ and the flow field is initialized with

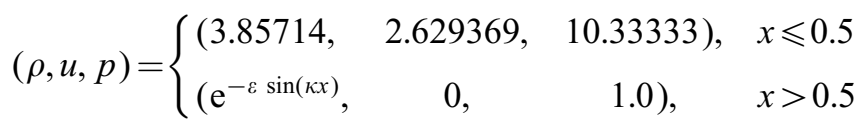

where $\varepsilon$ and $\kappa$ are the amplitude and the wavenumber of the entropy wave before the shock. The amplitude and wavenumber of amplified wave after the shock can be obtained from a linear analysis [19]. In our numerical experiments, we vary the wavenumber of the pre-shock entropy wave while keep its amplitude unchanged. As a result, the amplitude of the post-shock entropy wave will also be a constant, i.e. 0.08690716 and the corresponding amplitude of the pre-shock entropy wave is $\varepsilon=0.01$.

In this problem, a large-amplitude high-frequency entropy wave is mixed with spurious oscillations. It is difficult to distinguish them clearly in numerical simulations. Potential methods designed for suppressing the spurious oscillation might also smear the high-frequency post-shock entropy wave. As the wavenumber $\kappa$ increases, the problem becomes extremely challenging [1]. Low-order shock-capturing schemes and even some popular high-order schemes encounter the difficulty in preserving the amplitude of the entropy wave due to excessive dissipation with a given mesh size. Therefore, a success shock capturing method should be able to suppress Gibbs' oscillation, capture the shock and preserve the entropy wave.

The computational domain is deployed with 800 grid points, and such a mesh is used in all the numerical tests except for further specified. First, we consider the case of $\kappa=13$. This is 
a good test case for a basic scheme as there are 20 grid points per entropy wavelength, which is sufficient for describing the wave if there is no shock. Such a case was found being slightly difficult for the fifth order WENO scheme [1]. A significant amplitude damping occurs and the mesh of $N=1200$ has to be used to maintain the amplitude of the entropy wave [1]. The result of the CFOR scheme is depicted in Figure 4(a). It is seen that the generated entropy wave spans fully over the strip bounded by two solid lines, showing excellent agreement with the linear analysis. The shock is exactly captured with a small frequency mismatch located at the shock front. Such a mismatch also occurs to the ENO and WENO schemes [1] due to the nature of discontinuity. The performance of the CFOR scheme is really remarkable for this case.

Next, we double the wavenumber, i.e. $\kappa=26$. The number of supporting grid points per generated entropy wavelength is 10 . It is a quite difficult case for low-order shock-capturing schemes and no available result is reported in the literature, to our knowledge. The CFOR result is plotted in Figure 4(b). Obviously, the compressed entropy wave is excellently resolved in the post-shock regime. There is no visible trace of excessive dissipation as the amplitude of the entropy wave reaches its full strength in the whole post-shock regime. As expected, the frequency mismatch near the shock front becomes more obvious because there is an enlarged difference in the wave frequencies before and after the shock.

We further increase the wavenumber $\kappa$ to 39 and plot the CFOR result in Figure 4(c). It is interesting to note that the compressed entropy wave peaks span to its full amplitude for only about half of its extrema over the post-shock regime. Analysis indicates that the presence of the under developed peaks is not due to excessive dissipation. Instead, it is due to the insufficient resolution in the plot. With a total of 800 grid points in the domain, there is less than 7 grid points per wavelength. Such a grid is not large enough to fully resolve all the extrema in the compressed entropy wave. This explains the suppressed extrema in the plot. Obviously, it is extremely difficult to capture shock on such a grid for any potential scheme. However, the CFOR scheme performs extremely well as shown in Figure 4(d), which is obtained by interpolating the CFOR result in Figure $4(\mathrm{c})$ to a denser grid $(N=1600)$. The interpolation is carried out by using the DSC interpolation scheme, i.e. the conjugate low-pass filter as given in Equation (3) with $q=0$. Apparently, the quality of this result is comparable with the case of $\kappa=26$. This confirms that the CFOR scheme works well for shock-capturing under a very small ratio of grid points and wavelength.

Finally, we consider two large wavenumbers, $\kappa=52$ and 65 , to further test the performance of the CFOR scheme. A mesh of 800 grid points means a ratio of less than 5 grid points per wavelength, which is too few for simultaneous shock capturing and high-frequency wave resolving. The CFOR scheme generates some small amplitude damping for $\kappa=52$ (which is not shown). Therefore, we increase the mesh size to $N=1600$ for these computations. With this mesh, there are 10 grid points in each generated wavelength for $\kappa=52$. The resolution in the post-shock regime is excellent as shown in Figure 4(e). As shown in Figure 4(f), results for $\kappa=65$ are also very good. However, there is a visible amplitude damping in the generated entropy waves. We noticed that the conjugate low-pass filter is activated more often in this computation than in previous cases. With the increase of wavenumber $\kappa$, the $r$ should also be increased to broaden the effective bandwidth so that high-frequency wave shall not suffer from too much numerical damping. Obviously, the present scheme is capable of distinguishing the spurious oscillations from the high-frequency oscillation of the entropy wave, so as to attain high resolution for normal physical properties and capture the shock. It is 


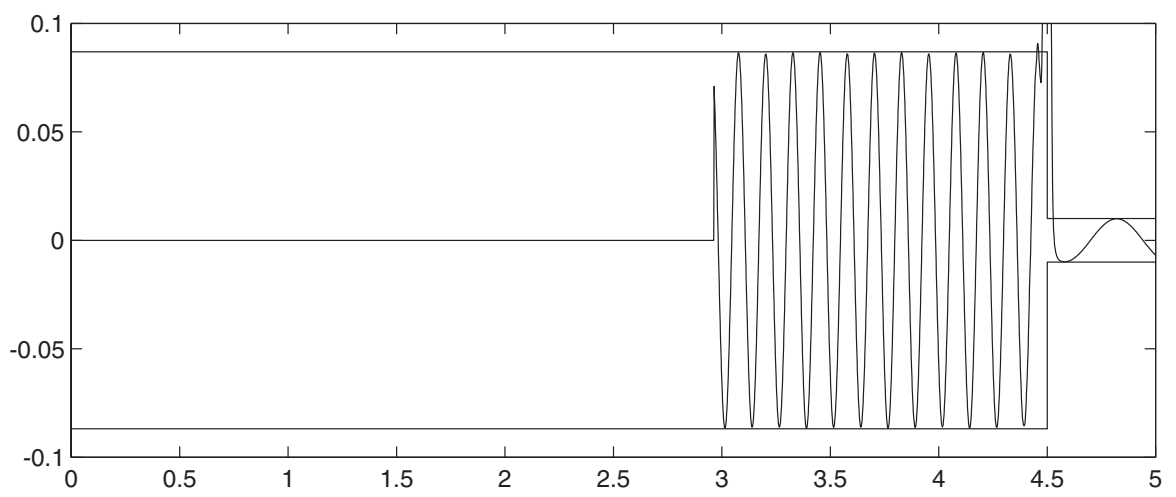

(a)

$\kappa=13, N=800$

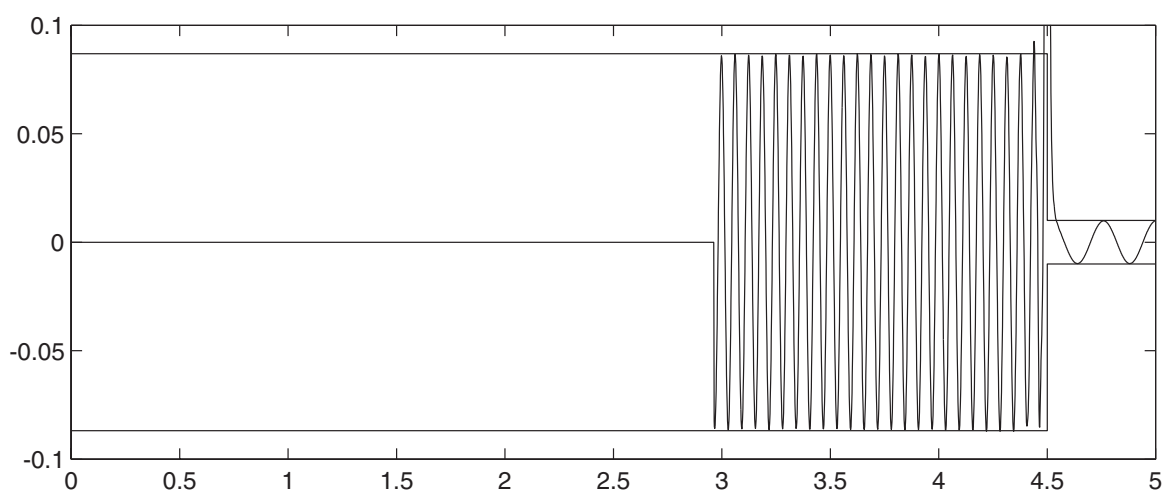

(b)

$\kappa=26, N=800$

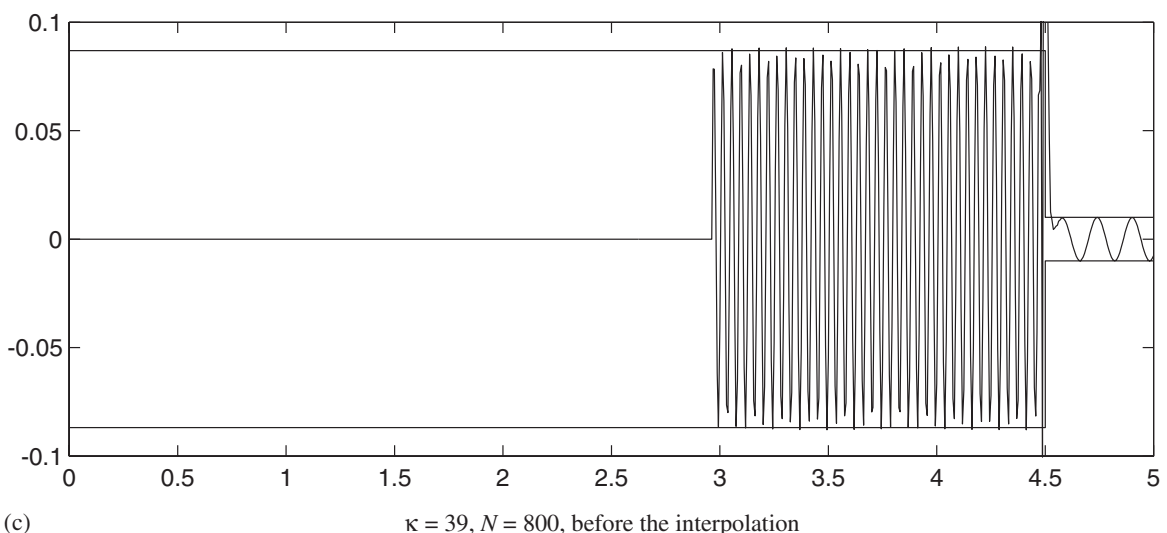

(c) $\kappa=39, N=800$, before the interpolation

Figure 4. Shock-entropy wave interaction: (a) $\kappa=13, N=800$; (b) $\kappa=26, N=800$; (c) $\kappa=39, N=800$, before the interpolation; (d) $\kappa=39, N=800$, after the interpolation; (e) $\kappa=52, N=1600$ and (f) $\kappa=65, N=1600$. 


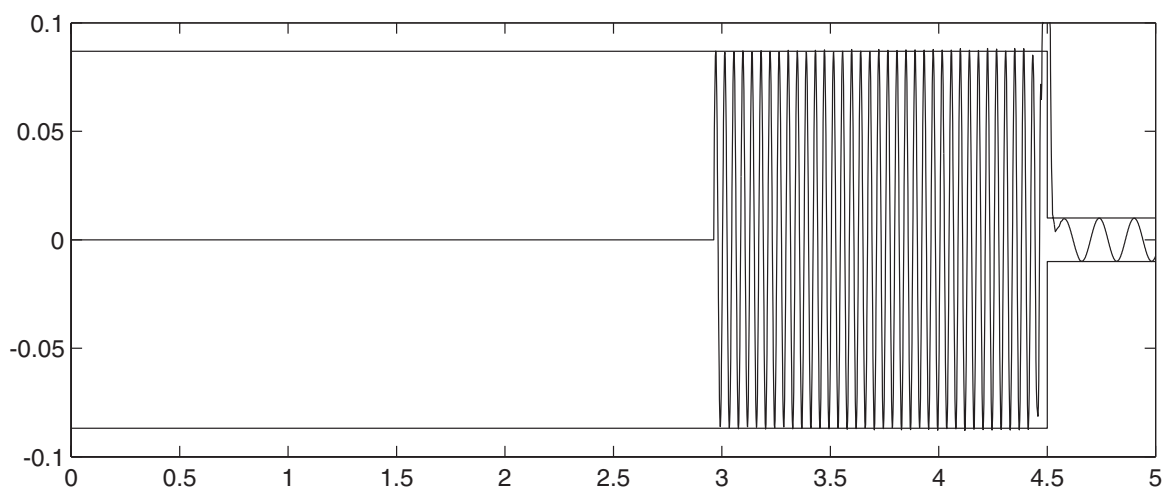

(d)

$\kappa=39, N=800$, after the interpolation

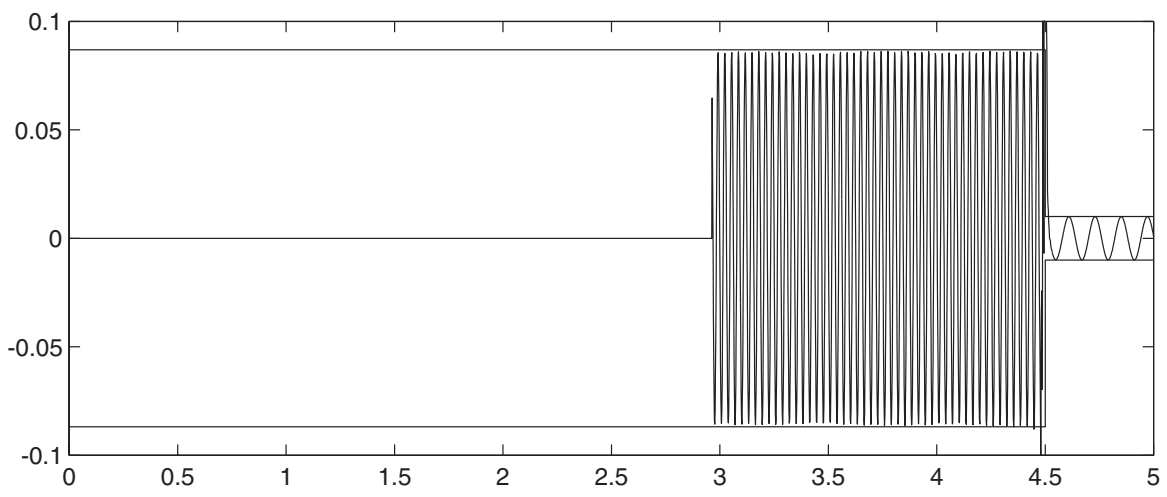

(e)

$$
\kappa=52, N=1600
$$

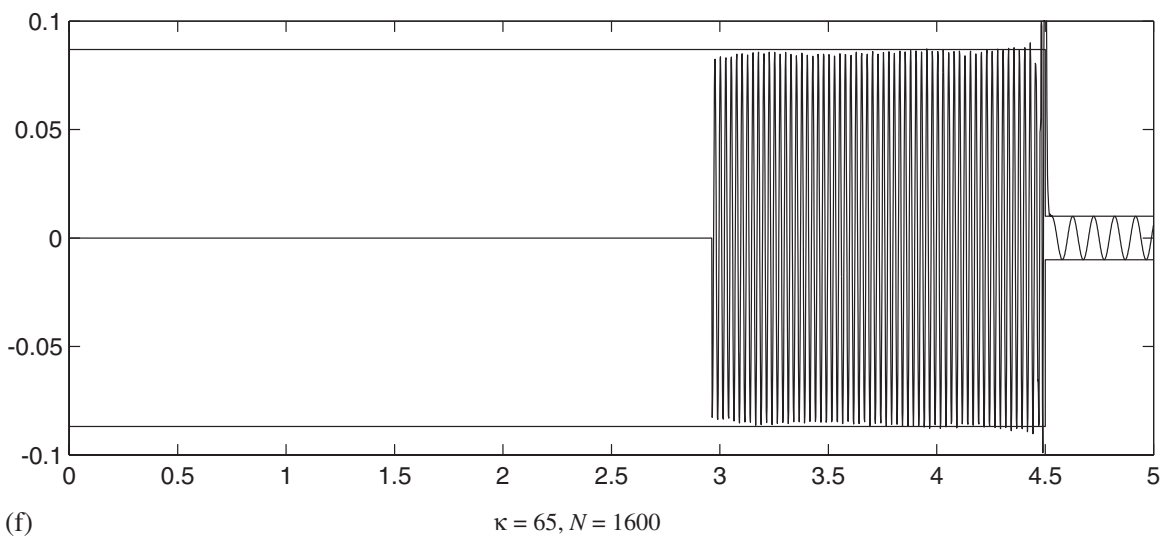

Figure 4. Continued. 
believed that the potential of the proposed CFOR scheme has been sufficiently demonstrated by these experiments.

\section{CONCLUSION}

The potential utility of a newly developed conjugate filter oscillation reduction (CFOR) scheme $[9,10]$ for the treatment of shock and high-frequency wave interaction is explored. The CFOR scheme is constructed based on the discrete singular convolution (DSC) algorithm, which is a practical approach for the numerical realization of singular convolutions. The essential idea of the CFOR scheme is to employ a conjugate low-pass filter to effectively remove the high-frequency errors (spurious oscillations) created by a set of high-pass filters, which are employed to discretize the spatial derivatives in the hyperbolic conservation laws or partial differential equations. The conjugate low-pass and high-pass filters are nearly optimal for shock-capturing and spurious oscillation suppressing in the sense that they are generated from the same expression and consequently have a similar order of regularity, approximation, effective frequency band and compact support.

The performance of the proposed scheme is examined by using an advection equation and two benchmark Euler systems. The accuracy and resolution of the DSC based algorithm is justified with the 1D advection equation. The first Euler system, free evolution of a 2D isentropic vortex [1,7], has an exact solution and its long-time evolution is a non-trivial task. The CFOR scheme provides higher-order accuracy for solving the problem and its performance is compared with many other schemes in the literature $[1,7]$. The other Euler system, the shockentropy wave interaction, is a difficult case due to its natural high-frequency oscillations in the compressed entropy wave, which is easily damped by the excessive numerical dissipation in most existing shock-capturing schemes. The problem becomes a severe challenge as the wavenumber increases. It is demonstrated that the CFOR provides some of the best solution ever available for this problem. The application of the CFOR scheme to more complicated problems and the adaptive optimization of the control parameters for conjugate filters are under consideration.

\section{ACKNOWLEDGEMENTS}

This work was supported by the National University of Singapore. The authors thank Prof. Pierre Sagaut and Prof. Eric Garnier, both at ONERA, for useful corresponding.

\section{REFERENCES}

1. Shu CW. Essentially non-oscillatory and weighted essentially non-oscillatory schemes for hyperbolic conservation laws. ICASE Report, No. 97-65, 1997.

2. Zienkiewicz OC, Nithiarasu P, Codina R, Vazquez M, Ortiz P. The characteristic-based-split procedure: an efficient and accurate algorithm for fluid problems. International Journal for Numerical Methods in Fluids 1999; 31:359-392.

3. Baumann CE, Oden JT. An adaptive-order discontinuous Galerkin method for the solution of the Euler equations of gas dynamics. International Journal for Numerical Methods in Engineering 2000; 47:61-73.

4. Lee S, Lele SK, Moin P. Interaction of isotropic turbulence with shock waves: effect of shock strength. Journal of Fluid Mechanics 1997; 340:225.

5. Garnier E, Mossi M, Sagaut P, Comet P, Deville M. On the use of shock-capturing scheme for large-eddy simulation. Journal of Computational Physics 2001; 153:273. 
6. Engquist B, Lötstedt P, Sjögreen B. Nonlinear filters for efficient shock computation. Mathematics of Computation 1989; 54:509-537.

7. Garnier E, Sagaut P, Deville M. A class of explicit ENO filters with application to unsteady flows. Journal of Computational Physics 2001; 170:184-204.

8. Lele SK. Compact finite difference schemes with spectral-like resolution. Journal of Computational Physics 1992; 103:16.

9. Wei GW, Gu Y. Conjugated filter approach for solving Burgers equation. Journal of Computational and Applied Mathematics 2002; 149:439-456.

10. Gu Y, Wei GW. Conjugated filter approach for shock capturing. Communications in Numerical Methods in Engineering 2003; 19:99-110.

11. Wei GW. Discrete singular convolution for the solution for the Fokker-Planck equations. Journal of Chemical Physics 1999; 110:8930-8942.

12. Wei GW. Wavelets generated by using discrete singular convolution kernels. Journal of Physics A, Mathematical and General 2000; 33:8577-8596.

13. Wei GW. A new algorithm for solving some mechanical problems. Computational Methods in Applied Mechanics and Engineering 2001; 190:2017-2030.

14. S. Ghosal. An analysis of numerical errors in large-eddy simulations of turbulence. Journal of Computational Physics 1996; 125:187.

15. Fedioun I, Lardjane N, Gökalp I. Revisiting numerical errors in direct and large eddy simulations of turbulence: physical and Fourier spaces analysis. Journal of Computational Physics 2001; 174:816.

16. Bao G, Wei GW, Zhou AH. Analysis of regularized Whittaker-Kotel'nikov-Shannon sampling expansion, preprint.

17. Vichenevetsky R, Bowles JB. Fourier Analysis of Numerical Approximations of Hyperbolic Equations. SIAM, Philadelphia, 1982

18. Yang SY, Zhou YC, Wei GW. Comparison of discrete singular convolution algorithm and the Fourier pseudospectral method for solving partial differential equations. Computer Physics Communications 2002; 143:113.

19. McKenzie JF, Westphal KO. Interaction of linear waves with oblique shock waves. Physics of Fluids 1968; 11:2350-2362. 